\title{
Materials Issues for the Catastrophic Degradation og High Power Laser Diodes
}

\author{
J. Souto, J.L. Pura, J. Jiménez* \\ GdS Optronlab, Universidad de Valladolid, Ed. Lucia, Paseo de Belén 19, 47011 Valladolid, Spain \\ ( ${ }^{*}$ jimenez@fmc.uva.es)
}

\begin{abstract}
In this work we investigate the catastrophic optical damage (COD) of GaAS based quantum well (QW) laser diodes under the optics of the role played by the physical properties of the materials forming the active parts of the lasers in the degradation process, taking account of their nanoscale dimensionality. High power laser diodes under operation can experiment local heating phenomena that lead to device failure. A thermo-mechanical model has been set up to study the mechanism of degradation. The incidence of the local heating in the degradation is tightly related to the physical properties of the active region of the laser; in particular, the thermal conductivity, the optical absorption, the thermal expansion coefficients, and the mechanical strength, play a crucial role in the degradation process. Furthermore, the low dimensionality of the layers has a strong influence on those properties.
\end{abstract}

Index Terms - catastrophic degradation, high-power laser diodes, mechanical strength, nanoscale, thermal conductivity, thermal stress

\section{INTRODUCTION}

The catastrophic optical damage (COD) is the sudden drop of the optical power output after hours of regular operation. The increasing request of optical power is limited by the COD. It is well known that the threshold for COD has been pushed up by the improvement of the epitaxial growth, mirror passivation, laser structure and packaging;. This degradation process is usually described as a thermal runaway process, in which the optical feedback leads the active zone of the laser to melt, [1,2]. The post morten analysis of the lasers reveals the existence of dark line defects (DLDs) along the laser cavity[3]. Fig.1 shows a cathodoluminescence (CL) image of the DLD in both the QW and the n-type guide layer of $980 \mathrm{~nm}$ single mode laser diode. These DLDs can be detected at the front mirror facet, but as the passivation and facet structures were improved the DLDs appear inside the laser cavity far from the facets. The dark line defects (DLDs) are aligned along the laser cavity, suggesting that they follow the optical field, instead of being related to a specific crystallographic direction. The propagation of the DLDs along the cavity is guided by the laser radiation, which is absorbed in tiny regions of the QW where the transparency was lost. The absorption of light at this tiny spot produces a local heat source, giving local temperature gradients, which are governed by the energy absorbed and the heat dissipation rate, which is dominated by the thermal conductivity $[4,5]$. This is a crucial magnitude, as the low dimensionality of the QW and the presence of interfaces substantially reduce the usually considered bulk thermal conductivity figures. The result of the local heat accumulation is a locally inhomogeneous temperature distribution, Fig.2. This non-uniform temperature produces thermal stresses because of the different thermal expansions of the laser constituents [6]. Therefore, the impact of the optical (optical absorption), thermal (thermal conductivity), and mechanical (yield strength) properties of the materials forming the laser structure on the COD has to be studied. The laser is multilayer structure formed by layers of different composition and thickness, which influence the values of the above mentioned properties, and, therefore, the behaviour of the laser diode towards the degradation. As mentioned above, the local temperature increase is responsible for the local laser absorption, and the subsequent positive energy feedback. The thermal conductivity is tightly related to the laser structure, since it depends on the thickness and composition of the layers, and the quality of the interfaces, in particular, the thermal conductivity is dramatically reduced in the QW. The thermal stresses built-up by the temperature gradients are also dependent on the laser structure; One should answer about the possibility that the thermal stresses might produce the onset of plasticity, thus launching the COD process, [6]. Here again, one should consider that the mechanical strength cannot be associated with the bulk figures but one has to consider the low thickness of the QW and the guide layers, as well as the interfaces. The mechanical strength of a multilayer structure is severely affected by those factors [7]. It is well known that strained QWs present a higher resilience than lattice matched QW lasers, [8]. The relation between QW temperature, stress and mechanical strength is summarized in Fig.3.

Finally, the evolution of the laser structure, associated with the formation of defects, during the laser operation produces changes in the materials and therefore their properties evolve as 
well; in particular, the thermal conductivity decreases with the presence of defects [9], and one can claim it as the factor producing the thermal runaway associated with the COD [10].

All these aspects are studied herein on the bases of a thermomechanical model for the COD of high power laser diodes based on GaAs related compounds [6,10]. Also, one discuss about the role of the QW and the guide layers in the end of life of the laser. This is crucially related to the above mentioned thermomechanical aspects, and to the laser absorbing medium. The role of the laser structure, in particular strained QWs, and multi quantum wells (MQW) in the degradation is also considered.

\section{ACKNOWLEDGMENT}

This work was funded by the Spanish Government (Grant: ENE201456069-C4-4-R) and Junta de Castilla y León (VA293U13 and VA081U16).

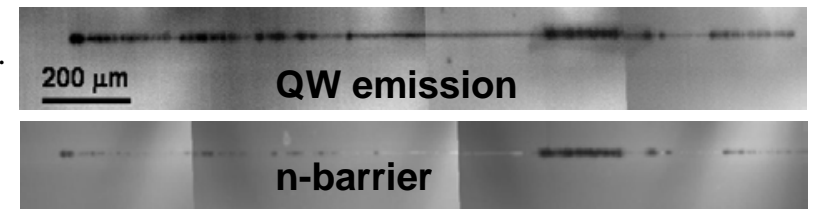

Fig.1. Cathodoluminescence images of the QW and n-barrier layer showing the DLDs along the cavity

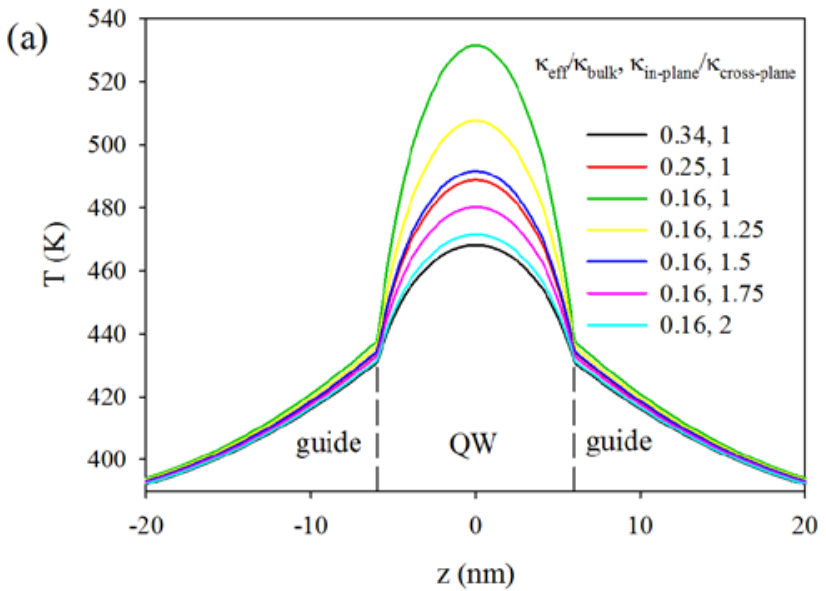

Fig.2. temperature profile along the epitaxial growth direction, for different effective thermal conductivities, showing the strong temperature gradient consequence of the low heat dissipation across the interfaces.

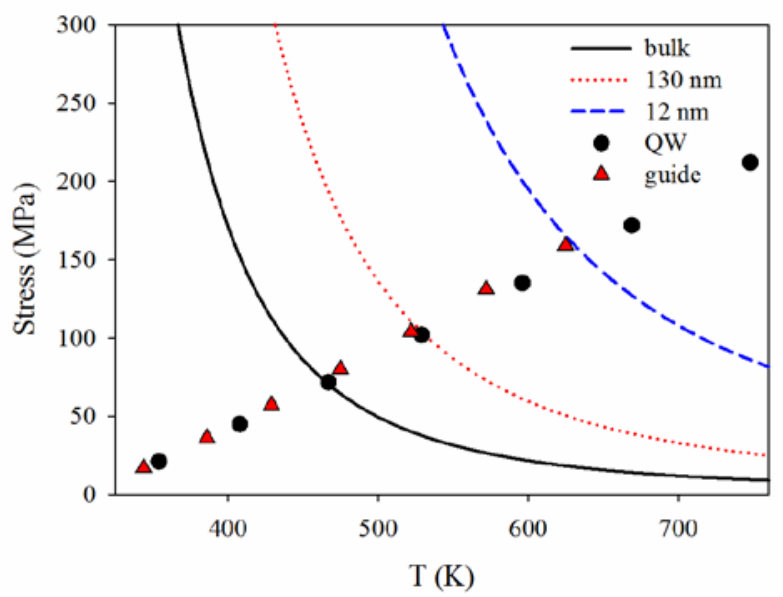

Fig.3. Stress vs QW temperature for the QW and the guide layer, in steps of absorption of $2 \mathrm{MW} / \mathrm{cm}^{2}$, the lines are the onsets of plasticity for bulk, the guide layer and the QW

\section{REFERENCES}

[1] J. Jiménez; Laser diode reliability: Crystal defects and degradation modes Comptes Rendus Phys. 4 663-73. 2003

[2] J.W.Tomm J W, M. Ziegler, M. Hempel and T. Elsaesser; Mechanisms and fast kinetics of the catastrophic optical damage (COD) in GaAs-based diode lasers Laser Photon. Rev. 5 422-41, 2011

[3] V. Hortelano, J. Anaya, J. Souto, J. Jiménez, J. Perinet, and F. Laruelle; Defect signatures in degraded high power laser diodes Microelectron. Reliab. 53 1501-5, 2013

[4] W. Nakwaski; Thermal model of the catastrophic degradation of highpower stripe-geometry $\mathrm{GaAs} /(\mathrm{AlGa}) \mathrm{As}$ double-heterostructure diode lasers J. Appl. Phys. 67 1659, 1990

[5] G. Chen and C.L. Tien; Facet heating of quantum well lasers J. Appl. Phys. 74 2167-74, 1993

[6] A. Martín-Martín, M. Avella, M.P. Iñiguez, J. Jiménez, M. Oudart and J. Nagle; Thermomechanical model for the plastic deformation in high power laser diodes during operation J. Appl. Phys. 106 73105, 2009

[7] P.E.Hopkins; Thermal transport across solid interfaces with nanoscale imperfections: Effects of roughness, disorder, dislocations, and bonding on thermal boundary conductance ISRN Mech. Eng 2013 682586, 2013

[8] R.G. Waters, R.J. Dalby, J.A. Baumann; J.L. De Sanctis, A.H. Shepard; Dark line resistant diode laser at $0.8 \mu \mathrm{m}$ comprising InGaAlAs strained QW; IEEE Photon. Technol. Lett, 3, 409-411, 1991

[9] A. Misra, J.P. Hirth and R.G. Hoagland; Length-scale-dependent deformation mechanisms in incoherent metallic multilayered composites Acta Mater. 53 4817-24, 2005

[10] J. Souto, J.L. Pura, J. Jimenez; Nanoscale effects on the thermal and mechanical properties of $\mathrm{AlGaAs} / \mathrm{GaAs}$ quantum well laser diodes: influence on the catastrophic optical damage; J. Phys. D Appl. Phys. 50 235101, 2017 